\title{
Role of Endoplasmic Reticulum Stress-Autophagy Axis in Severe Burn-Induced Intestinal Tight Junction Barrier Dysfunction in Mice
}

\author{
Yalan Huang ${ }^{1,2}$, Yu Wang ${ }^{3}$, Yanhai Feng ${ }^{2}$, Pei Wang ${ }^{2}$, Xiaochong He ${ }^{1}$, Hui Ren ${ }^{1 *}$ and \\ Fengjun Wang ${ }^{2 *}$
}

'School of Nursing, Third Military Medical University (Army Medical University), Chongqing, China, ${ }^{2}$ State Key Laboratory of Trauma, Burns and Combined Injury, Institute of Burn Research, Southwest Hospital, Third Military Medical University (Army Medical University), Chongqing, China, ${ }^{3}$ Department of Gastroenterology, Southwest Hospital, Third Military Medical University (Army Medical University), Chongqing, China

OPEN ACCESS

Edited by:

Stephen J. Pandol,

Cedars-Sinai Medical Center,

United States

Reviewed by:

Zhaolai Dai,

China Agricultural University, China

Darren Boehning,

University of Texas Health Science

Center at Houston, United States

*Correspondence:

Hui Ren

renhui_tmmu@163.com

Fengjun Wang

wangfj@tmmu.edu.cn

Specialty section:

This article was submitted to Gastrointestinal Sciences, a section of the journal Frontiers in Physiology

Received: 09 February 2019 Accepted: 29 April 2019

Published: 22 May 2019

Citation:

Huang $Y$, Wang $Y$, Feng $Y$, Wang $P$,

He $X$, Ren $H$ and Wang $F$ (2019)

Role of Endoplasmic Reticulum

Stress-Autophagy Axis in Severe

Burn-Induced Intestinal Tight Junction Barrier Dysfunction in Mice.

Front. Physiol. 10:606.

doi: 10.3389/fphys.2019.00606
Severe burn injury induces intestinal barrier dysfunction; however, the underlying mechanisms remain elusive. Our previous studies have shown that the intestinal epithelial tight junction (TJ) barrier dysfunction is associated with both endoplasmic reticulum (ER) stress and autophagy in severely burned mice, but the precise role of ER stress and autophagy in the burn-induced intestinal TJ barrier dysfunction needs to be determined. In this study, female C57/BL6 mice were assigned randomly to either sham burn or 30\% total body surface area (TBSA) full-thickness burn. The effects of ER stress and autophagy on the intestinal epithelial TJ barrier were validated by inducing or inhibiting both ER stress and autophagy in mice treated with sham burn or burn injury. The intestinal permeability, expression, and localization of TJ proteins, ER stress, and autophagy were assessed by physiological, morphological, and biochemical analyses. The results showed that inducing ER stress with tunicamycin or thapsigargin caused the activation of autophagy, the increase of intestinal permeability, as well as the reduction and reorganization of TJ proteins in the sham-burned mice, and aggravated the burn-induced activation of autophagy, increase of intestinal permeability, as well as the reduction and reorganization of TJ proteins. In contrast, inhibiting ER stress with 4-phenylbutyrate alleviated the burn-induced activation of autophagy, increase of intestinal permeability, as well as the reduction and reorganization of TJ proteins. In addition, inducing autophagy with rapamycin resulted in the increase of intestinal permeability, as well as the reduction and reorganization of TJ proteins in the sham-burned mice, and aggravated the burn-induced increase of intestinal permeability as well as the reduction and reorganization of TJ proteins. However, inhibiting autophagy with 3-methyladenine attenuated the burn-induced increase of intestinal permeability, as well as the reduction and reorganization TJ proteins. It is suggested that the ER stressautophagy axis contributes to the intestinal epithelial TJ barrier dysfunction after severe burn injury.

Keywords: burn injury, endoplasmic reticulum stress, autophagy, tight junction, intestinal barrier dysfunction 


\section{INTRODUCTION}

The intestinal epithelia form an important barrier between the intestinal mucosa and the luminal environment. The intestinal epithelial barrier, when intact, functions to resist the invasion of intraluminal bacteria, endotoxins, and other pathogens. The apical intercellular tight junctions (TJs) are essential for the intact paracellular barrier function. It is well known that increased permeability caused by the disruption of the intestinal epithelial TJ barrier is crucial for the development of intestinal inflammation (Arnott et al., 2000; Arrieta et al., 2009; Turner, 2009). We, along with other investigators, have found that severe burn injury directly causes the loss of intestinal barrier function and then leads to increased intestinal permeability (Deitch, 1990; Earley et al., 2015), both of which contribute to the development of systemic inflammation and multiple organ failure in severe burn injury (Magnotti and Deitch, 2005; Arrieta et al., 2009; Chen et al., 2012; Clark et al., 2017). Although the importance of burn-induced intestinal barrier disruption is recognized, the mechanisms of intestinal barrier dysfunction after severe burn injury remain poorly understood.

Endoplasmic reticulum (ER), as a membrane-bound organelle, plays a crucial role in folding of secreted and membrane proteins. ER stress triggers the unfolded protein response (UPR) to restore ER homeostasis toward reestablishing normal ER function through the mediation of three ER transmembrane sensors: activating the pancreatic ER stress kinase (PERK), inositol-requiring enzyme 1 (IRE1), and transcription factor 6 (ATF6) (Hosoi and Ozawa, 2009). Studies showed that excessive ER stress and impaired UPR signaling may cause inflammatory bowel diseases mainly through induction of intestinal epithelial cell apoptosis and intestinal proinflammatory response as well (Hiramatsu et al., 2015; Hosomi et al., 2015; Luo and Cao, 2015). Although we have found that the intestinal ER stress is significantly induced after severe burn injury (Huang et al., 2018), the contribution of ER stress to intestinal TJ barrier dysfunction is still not conclusive.

Autophagy is a catabolic process that degrades and recycles damaged organelles and misfolded proteins through the lysosomal machinery (Wirawan et al., 2012; Hale et al., 2013). Autophagy is active in the normal intestinal mucosa (Groulx et al., 2012); however, excessive autophagy and impaired autophagy participate in intestinal inflammation (Cooney et al., 2010; Wildenberg et al., 2012). We, along with other investigators, have shown that severe burn injury induced autophagy in some visceral organs such as the intestine (Huang et al., 2018), heart (Xiao et al., 2012), liver (Song et al., 2014), and lungs (Dong et al., 2017). The activation of autophagy has recently been reported to be associated with the epithelial barrier disruption in Caco-2 cell monolayers challenged with lipopolysaccharide (Feng et al., 2018). Accumulating data have revealed the bidirectional linkage between autophagy and ER stress (Yorimitsu et al., 2006; HoyerHansen and Jaattela, 2007; Qin et al., 2014), and ER stress activates autophagy via the inhibition of mTOR, the induction of Atg12 expression, or the activation of JNK (Ogata et al., 2006; Yorimitsu et al., 2006; Kouroku et al., 2007). Although our previous study has revealed that intestinal barrier dysfunction is accompanied by the activation of both ER stress and autophagy following severe burn injury (Huang et al., 2018), the exact role of ER stress and autophagy in the regulation of severe burn-induced intestinal barrier dysfunction remains unknown.

In this in vivo study, we demonstrate that severe burn induces ER stress and then activates autophagy in intestinal epithelial cells of mice. The ER stress-induced activation of autophagy results in the intestinal TJ barrier dysfunction following severe burn injury.

\section{MATERIALS AND METHODS}

\section{Ethics Statement}

The animal studies were approved by the Animal Care and Use Committee of the Third Military Medical University (Army Medical University), Chongqing, China. And all animal procedures were performed in adherence to protocols approved by the Ethics Committee of Southwest Hospital, Third Military Medical University (Army Medical University).

\section{ANIMAL MODEL AND PROCEDURES}

Healthy female C57BL6 mice (8-10 weeks old, weighing 20-25 g) were purchased from the Animal Center, Third Military Medical University (Army Medical University), fed with a standard rodent chow diet and watered and housed with a $12 \mathrm{~h}$ light/ dark cycle. All the animals were allowed to acclimate for 1 week prior to the experiment and randomly divided into 12 groups: control (sham burn), tunicamycin (Tm), thapsigargin ( $\mathrm{Tg}$ ), 4-phenylbutyrate (4-PBA), rapamycin (RAPA), 3-methyladenine (3-MA), burn, burn+Tm, burn+Tg, burn+4-PBA, burn+RAPA, and burn+3-MA. The mice in the indicated groups were intraperitoneally injected with tunicamycin $(1.0 \mathrm{mg} / \mathrm{kg}$; Calbiochem, MA, USA; D00176186) (Dong et al., 2015), thapsigargin (300 ng/kg; Sigma, St Louis, MO; 112M4011V) (Zhang et al., 2014), 4-PBA (80 mg/kg; Calbiochem, MA, USA; 2626840) (Ayala et al., 2012; Kim et al., 2013), rapamycin (4 mg/kg; Sigma, St Louis, MO; 2853724) (Wagner et al., 2012; Zhang et al., 2017a,b), or 3-methyladenine (15 mg/kg; Sigma, St Louis, MO; 026M4190V) (Zhang et al., 2014), respectively, $1 \mathrm{~h}$ before burn or sham burn. Meanwhile, the mice in the control or burn group just received the same volume of saline. A well-established method was used to induce a $30 \%$ fullthickness scald burn (Huang et al., 2018). Mice were anesthetized with $1 \mathrm{~g} / \mathrm{L}$ pentobarbital sodium (30 $\mathrm{mg} / \mathrm{kg}$ body weight i.p.), and the dorsal and lateral surfaces were shaved. The dorsum was immersed in $90^{\circ} \mathrm{C}$ water for $10 \mathrm{~s}$, and then lactated Ringer's solution ( $1 \mathrm{ml}$ ) was immediately administered intraperitoneally for resuscitation. The sham-burned mice received the same procedures except that the temperature of water was $37^{\circ} \mathrm{C}$. Finally, mice were housed in separate cages and anesthetized to monitor intestinal permeability at $6 \mathrm{~h}$ postburn. After that, the mice were immediately euthanized to collect the samples of ileum for histological, immunofluorescent, and immunoblot analysis, respectively. 


\section{Monitoring Intestinal Paracellular Permeability}

The intestinal paracellular permeability was determined as we described previously (Chen et al., 2012). Previous studies showed that intestinal permeability was sensitive in mouse ileum (Mazzon et al., 2002; Chen et al., 2012). Briefly, a laparotomy was performed under anesthesia, and a 5-cm segment of the ileum adjacent to the cecum was dissociated, with wellprotected superior mesenteric vessels. A 2-0 silk suture tied the isolated ileum at its bilateral end; $0.2 \mathrm{ml}$ of $0.1 \mathrm{~mol} / \mathrm{L}$ phosphate-buffered saline (PBS, pH 7.2) containing fluorescein isothiocyanate-labeled dextran (FITC-dextran, $20 \mathrm{mg} / \mathrm{ml}$ ) (Sigma, St. Louis, MO) was injected into the lumen. The blood sample was taken after $30 \mathrm{~min}$ and centrifuged at $4^{\circ} \mathrm{C}, 3,000 \times \mathrm{g}$, for $10 \mathrm{~min}$. The plasma was taken and diluted at 1:10 with PBS to measure the fluorescence intensity with a microplate reader (Varioskan Flash, Thermo Electron Corporation, Vantaa, Finland) with an excitation wavelength of $480 \mathrm{~nm}$ and an emission wavelength of $520 \mathrm{~nm}$. The concentrations of plasma FITC-dextran were calculated from a standard curve generated by serial dilution of FITC-dextran in PBS.

\section{Immunoblot Analysis}

Ileal mucosa scrapings were collected and homogenized in RIPA buffer. The lysate was centrifuged at $4^{\circ} \mathrm{C}, 15,000 \times g$, for $15 \mathrm{~min}$, and the supernatants were collected for protein expression assessment. Protein concentration was determined using the $R C D C$ protein assay kit (Bio-Rad, Hercules, CA). RC DC protein assay is a colorimetric assay for protein quantitation with all the functionality of the original DC protein assay. This assay is based on the Lowry assay but has been modified to be reducing-agent compatible as well as detergent compatible. Western blotting was performed as we reported (Huang et al., 2018); $30 \mathrm{mg}$ protein was loaded for each lane and then separated on SDS-PAGE gel $(6,8,10$, or $12 \%$ ) and transferred to a PVDF membrane (Millipore). Membranes were blocked using 5\% non-fat milk at room temperature for $1 \mathrm{~h}$ and then probed with the following primary antibodies at $4^{\circ} \mathrm{C}$ overnight: ZO-1 (1:1,000; Invitrogen, cat. no. 33-9100, CA, USA), occludin (1:1,000; Invitrogen, cat. no. 71-1500, CA, USA), claudin-1 (1:1,000; Invitrogen, cat. no. 37-4490, CA, USA), Bip (1:1,000; Cell Signaling, cat. no. 3177, MA, USA), CHOP (1:1,000; Cell Signaling, cat. no. 2895, MA, USA), XBP1 (1:1,000; abcam, cat. no. ab37152, MA, USA), LC3B (1:1,000; Sigma-Aldrich, cat. no. L7543, MO, USA), p62 (1:1,000; Sigma-Aldrich, cat. no. P0067, MO, USA), Beclin-1 (1:1,000; Sigma-Aldrich, cat. no. PRS3613, MO, USA), Atg5 (1:1,000; Sigma-Aldrich, cat. no. A0731, MO, USA), PI3 kinase p85 (1:1,000; Cell Signaling, cat. no. 4257, MA, USA), phospho-PI3 kinase p85/p55 (1:1,000; Cell Signaling, cat. no. 4228, MA, USA), Akt (1:1,000; Cell Signaling, cat. no. 4691, MA, USA), phospho-Akt (Ser473) (1:1,000; Cell Signaling, cat. no. 4060, MA, USA), mTOR (1:1,000; Cell Signaling, cat. no. 2983, MA, USA), phospho-mTOR (1:1,000; Cell Signaling, cat. no. 5536, MA, USA), and $\beta$-actin (1:5,000; Sigma-Aldrich, cat. no. A5316, MO, USA). After washing, membranes were incubated with peroxidase-conjugated secondary antibodies (Southern Biotech, Birmingham, AL, United States) for $1 \mathrm{~h}$ at room temperature. Finally, the blots were visualized with an enhanced chemiluminescence detection kit (GE Healthcare, Buckinghamshire, United Kingdom) and imaged with a ChemiDoc XRS system (Bio-Rad, Hercules, CA, United States). Quantity One software (Bio-Rad) was used for densitometric analysis.

\section{Immunofluorescence}

The immunofluorescence was performed as previously reported (Huang et al., 2018). Frozen sections of ileal tissue were fixed with $1 \%$ paraformaldehyde and then permeabilized in $1 \%$ Triton X-100 (Sigma, T9284). The nonspecific binding sites were blocked with $5 \%$ normal goat serum for $30 \mathrm{~min}$. Sections were stained with ZO-1 antibody at 1:100 and fluorescenceconjugated secondary antibodies: Alexa Fluor 488-conjugated goat anti-rabbit IgG antibody (Invitrogen) at 1:100 and Alexa Fluor 594-conjugated phalloidin (Invitrogen) at 1:40. The ileal tissues were viewed with a TCS SP5 laser confocal microscope (Leica, Germany) and analyzed using Leica LAS AF 2.3.0 software (Leica Microsystems, Germany).

\section{Transmission Electron Microscopy}

The ileal tissues were harvested and quickly fixed with $2.5 \%$ glutaraldehyde in $0.1 \mathrm{~mol} / \mathrm{L}$ PBS ( $\mathrm{pH} 7.4$ ) for $2 \mathrm{~h}$. Then the samples were fixed with $2 \%$ osmium tetroxide, dehydrated in a graded series of alcohol, and finally embedded in Epon 812. An ultramicrotome (Ultract N; Reichert-Nissei, Tokyo, Japan) was used to cut the samples for ultrathin sections. Collected sections were fixed with $2 \%$ uranyl acetate and lead citrate and then photographed under a transmission electron microscope (JEM-1400PLUS, Japan).

\section{Statistical Analysis}

SPSS 13.0 software was used for statistical analysis. The data are presented as mean \pm SEM. Statistical significance was calculated using one-way analysis of variance (ANOVA) and was set at $p<0.05$. All reported significance levels represent two-tailed $p$ 's.

\section{RESULTS}

\section{Severe Burn-Induced Intestinal Epithelial ER Stress Promotes the Activation of Autophagy via Inhibition of the PI3K/AKT/mTOR Pathway}

In our previous study, we have found that severe burn injury induced the activation of ER stress in intestinal mucosa, which was accompanied by the activation of autophagy (Huang et al., 2018). However, the exact relationship between ER stress and autophagy in severe burn injury is not clear. To determine the association between ER stress and autophagy in intestinal mucosa, we detected the level of autophagy after the treatment of ER stress inducers ( $\mathrm{Tm}$ and $\mathrm{Tg}$ ) or inhibitor (4-PBA) in 
mice with or without burn injury. We firstly investigated the effect of $\mathrm{Tm}, \mathrm{Tg}$, and 4-PBA on ER stress in burned or shamburned mice. As shown in Figure 1A, the expression levels of the ER stress-related proteins Bip, XBP1, and CHOP were all enhanced after burn injury or Tm or Tg treatment compared with control. Both $\mathrm{Tm}$ and $\mathrm{Tg}$ further aggravated the severe

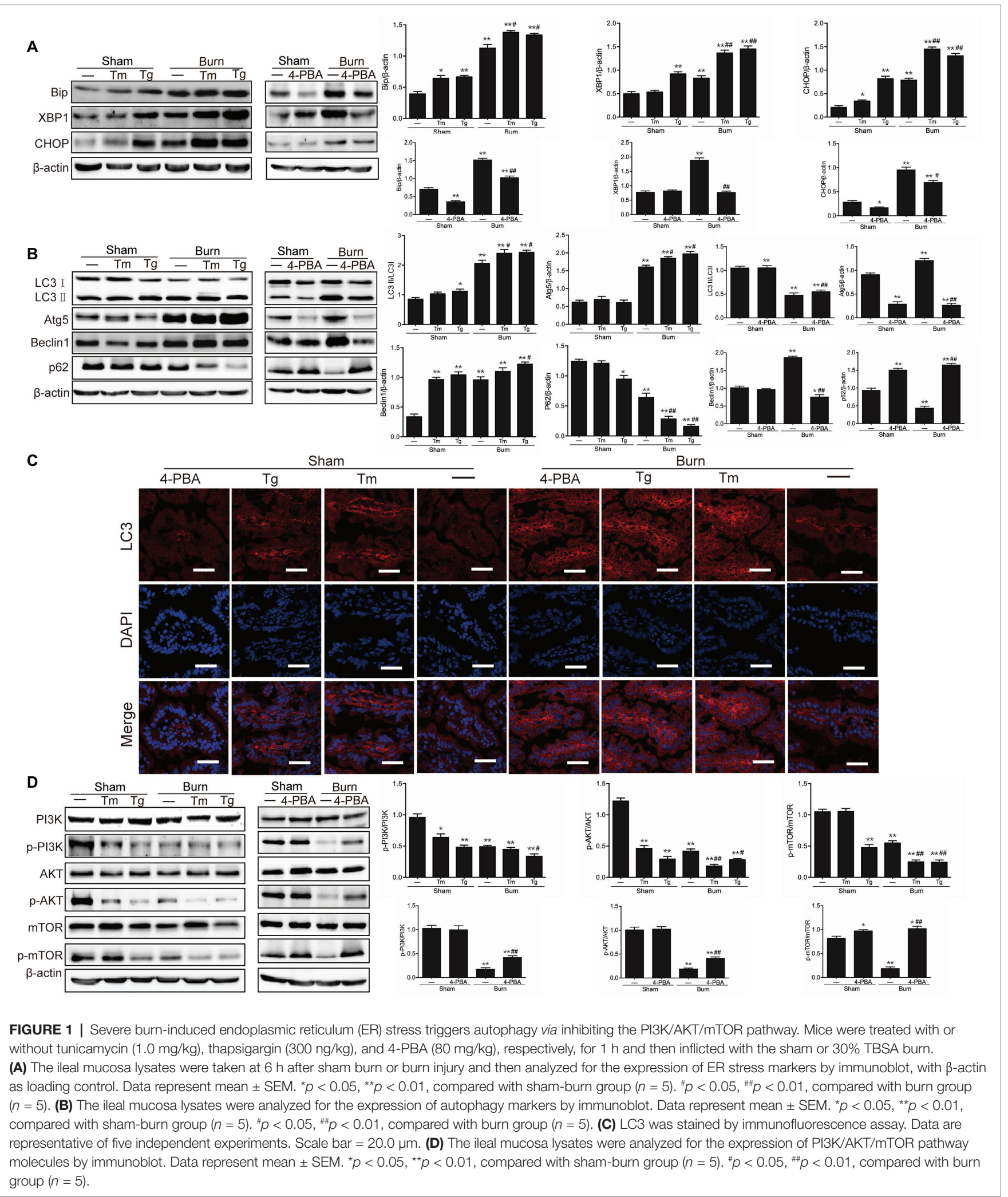


burn-induced upregulation of Bip, XBP1, and CHOP. However, 4-PBA, a chemical chaperone that was reported to inhibit ER stress in both cell lines and animals (Ozcan et al., 2006; Ozean et al., 2008), decreased the protein levels of Bip, XBP1, and $\mathrm{CHOP}$ in severely burned mice, also as illustrated in Figure 1A.

We next determined the effect of ER stress on autophagy in intestinal epithelia. As shown in Figure 1B, the severe burn-induced increase of LC3-II/LC3-I, Atg5, and Beclin 1 were all significantly aggravated by inducing ER stress with $\mathrm{Tm}$ and $\mathrm{Tg}$, the ER stress inducers. Conversely, 4-PBA, an ER stress inhibitor, blocked the severe burn-induced increase of LC3-II/LC3-I, Atg5, and Beclin 1. Consistently, the severe burn-induced decrease of p62 was significantly aggravated by $\mathrm{Tm}$ or $\mathrm{Tg}$ treatment but reversed by inhibiting ER stress with 4-PBA.

We also detected LC3 by an immunofluorescent staining assay in intestinal epithelia. As illustrated in Figure 1C, treatment of severely burned mice with $\mathrm{Tm}$ or $\mathrm{Tg}$ aggravated the severe burn-induced increase of total LC3 protein. However, the severe burn-induced increase of total LC3 protein was significantly reduced by 4 -PBA treatment. Taken together, these data suggest that induction of ER stress promotes the activation of autophagy in the intestinal epithelia of mice suffering from severe burn injury.

Considering the reports that the PI3K/AKT/mTOR signaling pathway plays an important role in the interplay between ER stress and autophagy, we further determined whether the PI3K/ AKT/mTOR signaling pathway participates in the autophagy activation by severe burn-induced ER stress in intestinal epithelia. As shown in Figure 1D, the expression of phosphorylated PI3K, AKT, and mTOR was remarkably reduced by the treatment of Tm or Tg to induce ER stress but markedly increased by 4-PBA treatment to inhibit ER stress in mice with severe burn. These results indicate that the suppression of the PI3K/AKT/mTOR signaling pathway is involved in the autophagy activation by severe burn-induced ER stress in intestinal epithelia.

\section{Activation of Endoplasmic Reticulum Stress is Required for Intestinal Tight Junction Barrier Dysfunction in Severely Burned Mice}

Based on our previous study that severe burn-induced intestinal barrier dysfunction was accompanied by the activation of ER stress (Huang et al., 2018), we sought to determine the contribution of ER stress to the severe burn-induced intestinal epithelial TJ barrier dysfunction. As revealed in Figure 2A, the intestinal paracellular permeability to $4.4 \mathrm{kDa}$ FITCdextran was significantly increased following severe burn injury. More importantly, the severe burn-induced increase of intestinal paracellular permeability was remarkably aggravated by $\mathrm{Tm}$ or $\mathrm{Tg}$ treatment but distinctly attenuated by 4 -PBA treatment.

To further investigate the role of ER stress activation in the intestinal barrier dysfunction, the damage of the intestinal mucosa was examined by hematoxylin and eosin staining. As shown in Figure 2B, in control mice, the intestinal epithelia were intact, and the villi were in good order. However, in the intestinal mucosa of burned mice, the epithelial cells were exfoliated and accompanied by exposed lamina propria, necrosis, and inflammatory cell infiltration. Notably, the severe burninduced histological damages were aggravated by $\mathrm{Tm}$ or $\mathrm{Tg}$ treatment and dramatically alleviated by 4 -PBA.

Considering the critical roles of $\mathrm{TJ}$ proteins in the regulation of intestinal epithelial barrier function, we then investigated whether inducing or inhibiting ER stress affected TJ protein expression and ZO-1 localization in severely burned mice. As shown in Figure $2 \mathrm{C}, \mathrm{Tm}$ or $\mathrm{Tg}$ treatment caused a remarkable decline in the expression of ZO-1 and claudin-1 in mice with or without burn injury. The protein level of occludin was significantly reduced by $\mathrm{Tm}$ or $\mathrm{Tg}$ treatment in severely burned mice. However, 4-PBA treatment reversed the severe burninduced decrease of ZO-1, occludin, and claudin-1.

$\mathrm{ZO}-1$ is indispensable in the maintenance of the intestinal leaky pathway and the regulation of paracellular permeability of the TJ, and its defects directly result in the failure of $\mathrm{TJ}$ formation (Umeda et al., 2006). Our previous studies have demonstrated that $\mathrm{ZO}-1$, as an important $\mathrm{TJ}$ protein in the maintenance and regulation of the intestinal leaky pathway, has been relocalized after severe burn injury (Chen et al., 2012; Huang et al., 2018). Thus, we further sought to assess the influence of ER stress on morphological changes of the ZO-1 protein in severely burned mice by immunofluorescent assay. As illustrated in Figure 2D, ZO-1 (shown as red) was localized to the epithelial TJs and can be seen as a series of ordered bright red spots at the apical junctions in the control group. Treatment of mice with $\mathrm{Tm}$ or $\mathrm{Tg}$ resulted in ZO-1 relocalization and worsened the severe burninduced reorganization of ZO-1, which can be seen as a loss of bright red spots at the apical junctions. However, treatment of mice with 4-PBA did not obviously affect the morphological distribution of ZO-1 in sham-burned mice, but evidently alleviated the relocalization of ZO-1 in severely burned mice.

\section{Autophagy Activation Is Involved in Intestinal Tight Junction Barrier Dysfunction Induced by Severe Burn Injury}

In our previous study, we have found that the severe burninduced TJ barrier dysfunction was accompanied by the autophagy activation in the ileum of burned mice (Huang et al., 2018). Thus, we sought to determine whether the activation of autophagy was involved in the intestinal TJ barrier dysfunction caused by severe burn injury. First, we treated burned or sham-burned mice with rapamycin, an mTOR inhibitor and classical autophagy inducer, or 3-MA, an autophagy inhibitor suppressing the activity of class III PI3K, to trigger or suppress autophagy activation, respectively. As shown in Figure 3A, treatment of burned or sham-burned mice with rapamycin resulted in a striking increase in LC3П/LC3I ratio and the expression of both Atg5 and Beclin 1, and a decrease in p62 expression in the intestinal epithelia. On the contrary, 3-MA treatment caused a marked decrease in LC3 /LC3I ratio and the expression of both Atg5 and Beclin 1, and elevation of p62 expression in the intestinal epithelia of burned mice. Consistent with these 


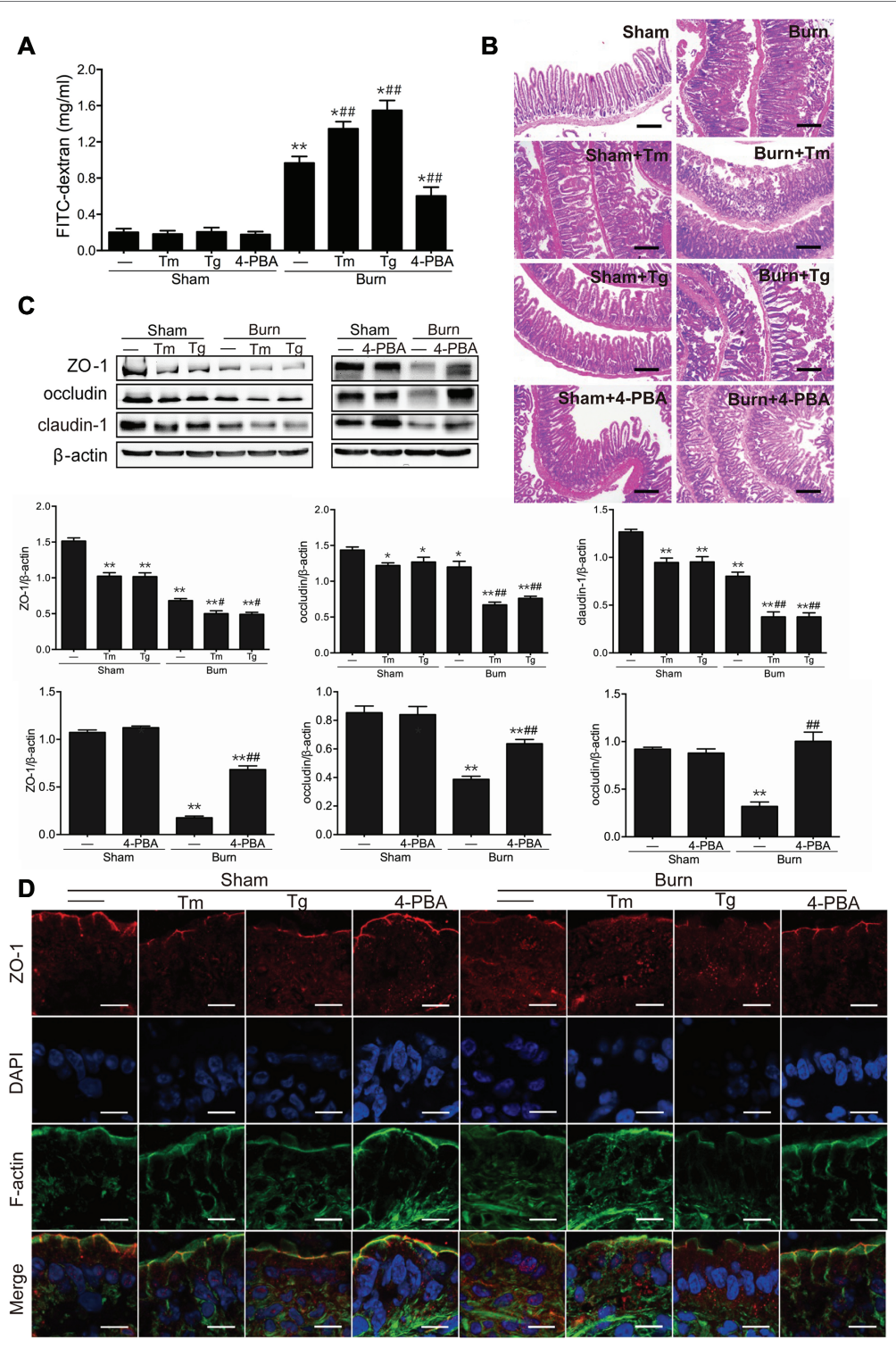

FIGURE 2 | Severe burn-induced intestinal epithelial tight junction (TJ) barrier dysfunction is aggravated by ER stress inducers and alleviated by ER stress inhibitor. Mice were treated as described in Figure 1. (A) Intestinal permeability to $4.4 \mathrm{kDa}$ FITC-dextran was measured at $6 \mathrm{~h}$ after sham burn or burn injury as described in "Materials and Methods." Data represent mean \pm SEM. ${ }^{*} p<0.05,{ }^{* *} p<0.01$, compared with sham-burn group $(n=5)$. ${ }^{\#} p<0.05$, ${ }^{\# \#} p<0.01$, compared with burn group $(n=5)$. (B) Hematoxylin and eosin staining of distal ileal segments was performed at $6 \mathrm{~h}$ following sham burn or $30 \%$ TBSA burn. Data are representative of five independent experiments. Scale bar $=200 \mu \mathrm{m}$. (C) Immunoblot was performed to determine the expression of ZO-1, occludin, and claudin-1, with $\beta$-actin as loading control. Data represent mean \pm SEM. ${ }^{*} p<0.05$, ${ }^{* *} p<0.01$, compared with sham-burn group $(n=5)$. ${ }^{*} p<0.05$, ${ }^{\# \#} p<0.01$, compared with burn group $(n=5)$. (D) ZO-1 was stained by immunofluorescence assay. Data are representative of five independent experiments. Scale bar $=20.0 \mu \mathrm{m}$.

results, rapamycin treatment significantly increased the number of autolysosome and autophagosomes in the intestinal epithelial cells of burned or sham-burned mice, whereas 3-MA treatment remarkably decreased the number of autolysosome and autophagosomes in mice inflicted with severe burn injury, as illustrated in Figure 3B. In addition, as shown in Figure 3C, the protein expression of phosphorylated PI3K, phosphorylated $\mathrm{AKT}$, and phosphorylated mTOR were all remarkably reduced by the treatment of rapamycin but markedly increased by 3-MA.

We then evaluated the effect of autophagy activation on epithelial $\mathrm{TJ}$ barrier function in the ileum of mice with or without severe burn. As revealed in Figure 4A, inducing autophagy with rapamycin significantly elevated paracellular permeability to FITC-dextran in sham-burned mice and distinctly exacerbated the severe burninduced increase of paracellular permeability in burned mice. In contrast, inhibiting autophagy with 3-MA had no significant effect on paracellular permeability but markedly dampened severe burninduced increase of paracellular permeability in the ileum of mice suffering from severe burn injury. Moreover, we further determined the influence of autophagy activation on severe burn-induced histological damage of intestinal mucosa. As illustrated in Figure 4B, the damage of ileal mucosa was obviously in burned mice when 
A

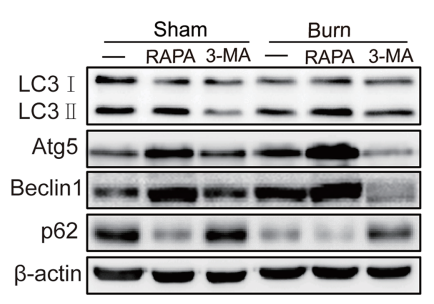

B
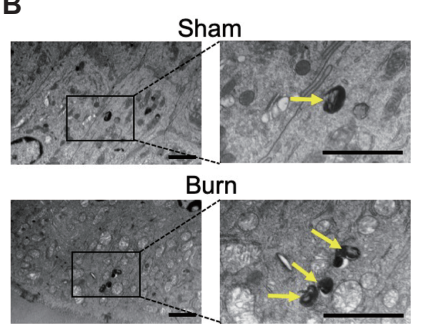

C

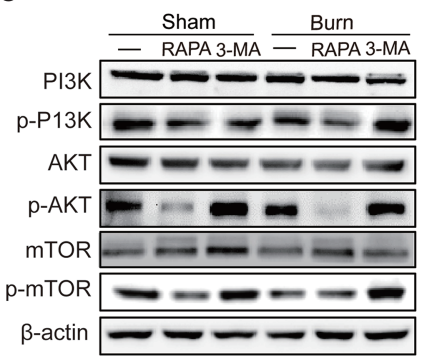

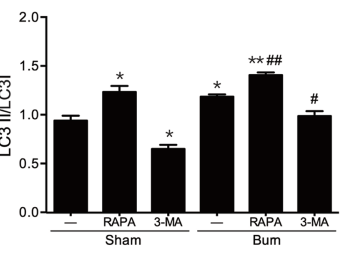
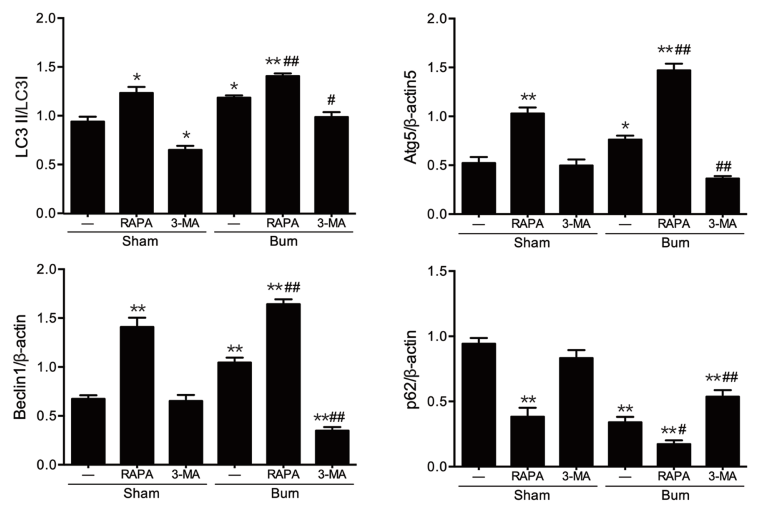

Sham+RAPA

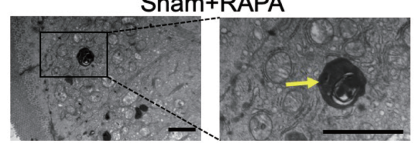

Burn+RAPA
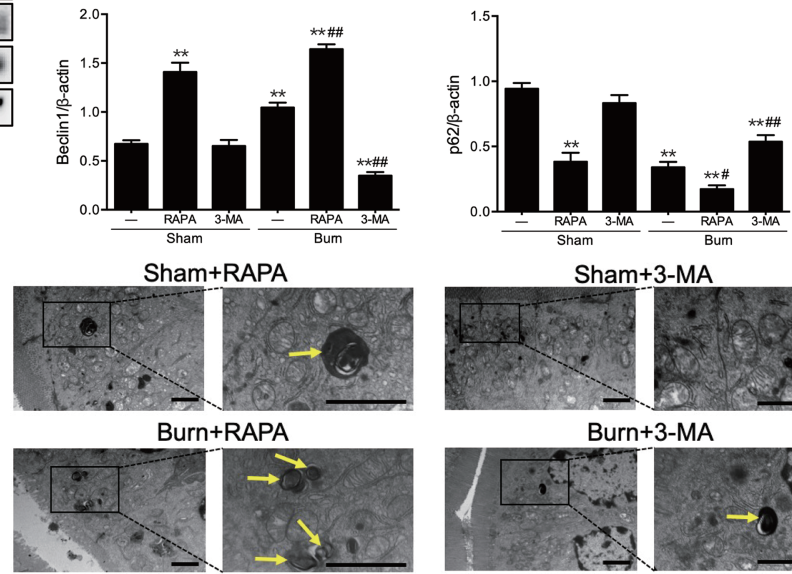

Sham+3-MA

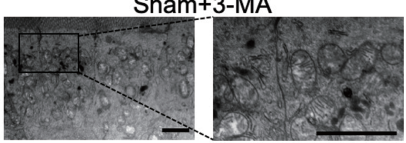

Burn+3-MA
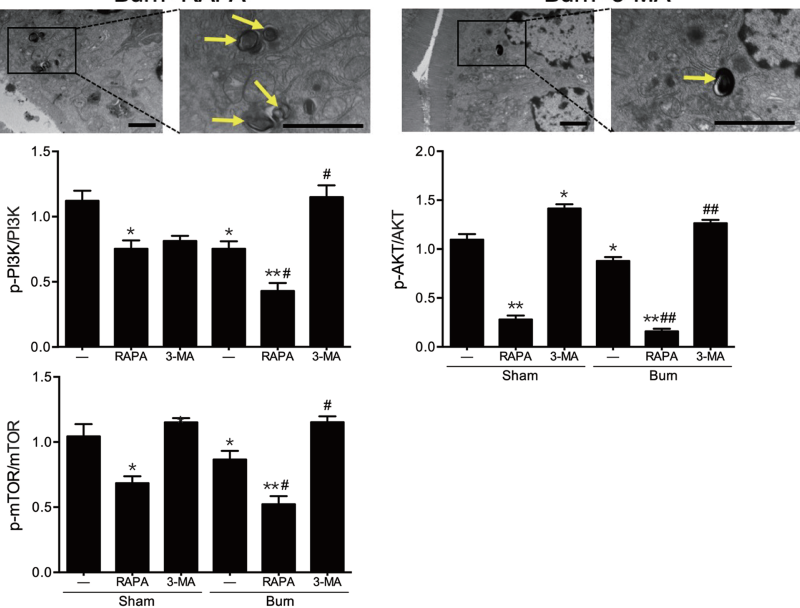

FIGURE 3 | Severe burn-induced autophagy activation is exacerbated by autophagy inducer and suppressed by autophagy inhibitor. Mice were treated with or without rapamycin (4 mg/kg) and 3-methyladenine (15 mg/kg), respectively, for $1 \mathrm{~h}$ and then given the sham or 30\% TBSA burn. (A) The ileal mucosa lysates were taken at $6 \mathrm{~h}$ after sham burn or burn injury and then analyzed for the expression of autophagy markers by immunoblot, with $\beta$-actin as loading control. Data represent mean \pm SEM. ${ }^{*} p<0.05,{ }^{* *} p<0.01$, compared with sham-burn group $(n=5)$. ${ }^{*} p<0.05$, ${ }^{\# \#} p<0.01$, compared with burn group ( $n=5$ ). (B) Representative autophagosome (yellow arrow) images of intestinal epithelial cells from sham-burned or burned mice under transmission electron microscopy (TEM). Scale bar $=2.0 \mu \mathrm{m} . n=5$. (C) The ileal mucosa lysates were analyzed for the expression of PI3K/AKT/mTOR pathway molecules by immunoblot. Data represent mean \pm SEM. ${ }^{*} p<0.05,{ }^{* *} p<0.01$, compared with sham-burn group $(n=5)$. ${ }^{*} p<0.05,{ }^{\# \#} p<0.01$, compared with burn group $(n=5)$.

compared to the sham-burned mice. Notably, rapamycin treatment remarkably exasperated the burn-induced damage of ileal mucosa. In contrast, treatment of mice with 3-MA did not affect the histological morphology of ileal mucosa in sham-burned mice, but evidently alleviated the severe burn-induced histological damage of ileal mucosa. These results imply that activation of autophagy aggravates the intestinal epithelial barrier dysfunction induced by severe burn, whereas inhibition of autophagy attenuates the severe burn-induced intestinal epithelial barrier dysfunction.

Having confirmed that the autophagy activation is involved in severe burn-induced intestinal barrier dysfunction, we next investigated the effect of autophagy activation on the expression of TJ proteins in the ileal mucosa of mice inflicted with or without burn injury. As shown in Figure 4C, rapamycin treatment significantly reduced the expression of $\mathrm{ZO}-1$ and claudin-1 in sham-burned mice and distinctly exacerbated the severe burninduced decrease of ZO-1, occludin, and claudin-1 expression in burned mice. On the contrary, 3-MA treatment did not remarkably alter the expression of ZO-1, occludin, and claudin-1 in sham-burned mice, but almost completely blocked the severe burn-induced decrease of ZO-1, occludin, and claudin-1 expression in burned mice. Moreover, the effect of autophagy activation on the distribution and localization of ZO-1 in the ileum was examined by immunofluorescent assay coupled with confocal microscopy. As demonstrated in Figure 4D, ZO-1 exhibited a series of red spots that were bright and ordered at the apical junctions in sham-burned mice. In contrast, the burned mice showed disorganized and random ZO-1 staining. Treatment of mice with rapamycin caused relocalization of $\mathrm{ZO}-1$ in sham-burned mice and worsened the severe burn-induced ZO-1 reorganization, which 


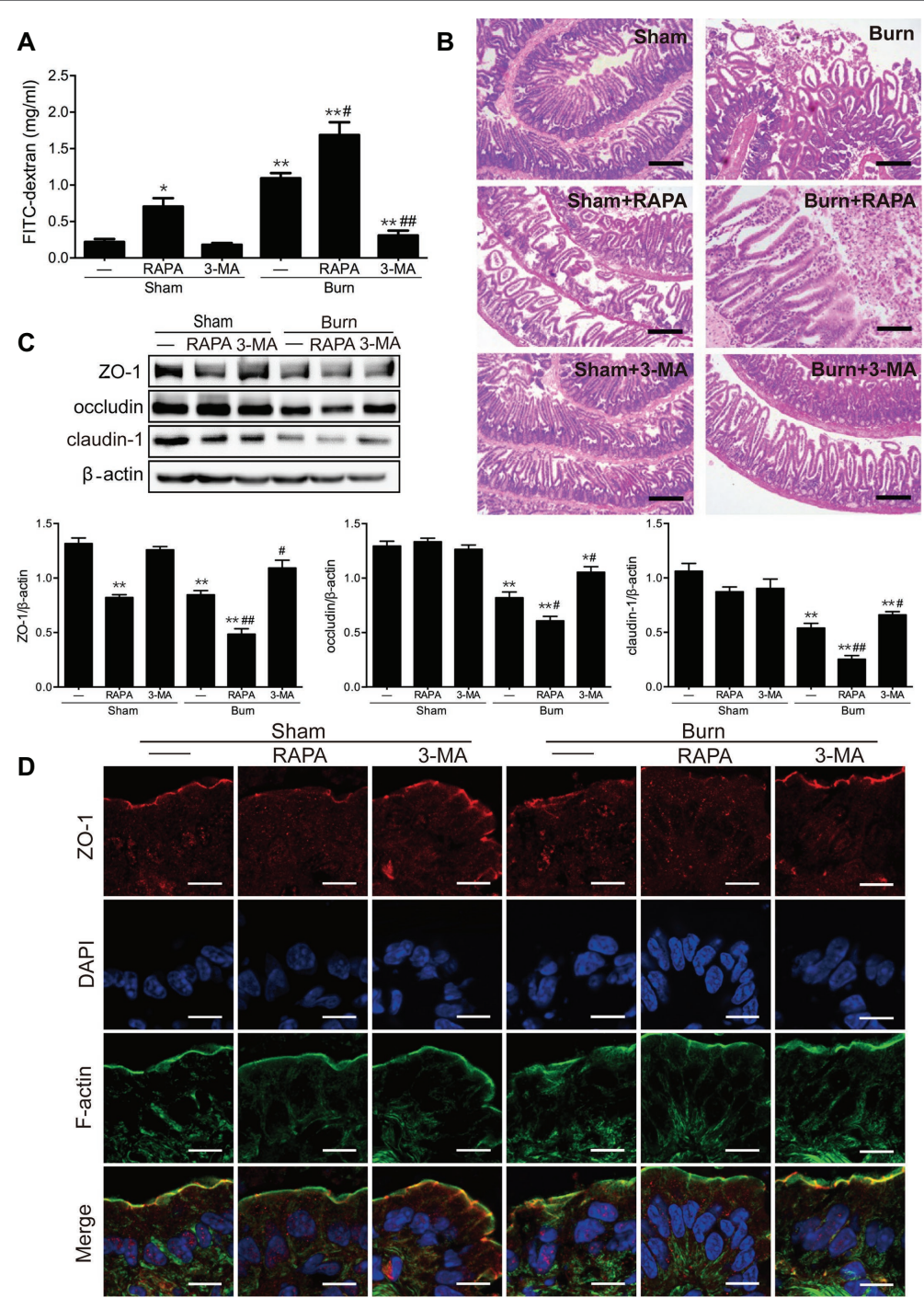

FIGURE 4 | Severe burn-induced intestinal epithelial TJ barrier dysfunction is exacerbated by rapamycin and ameliorated by 3-MA. Mice were treated as described in Figure 3. (A) Intestinal permeability to $4.4 \mathrm{kDa}$ FITC-dextran was measured at $6 \mathrm{~h}$ after sham burn or burn injury as described in "Materials and Methods." Data represent mean \pm SEM. ${ }^{*} p<0.05,{ }^{\star *} p<0.01$, compared with sham-burn group $(n=5) .{ }^{*} p<0.05$, ${ }^{\# *} p<0.01$, compared with burn group ( $n=5$ ). (B) Hematoxylin and eosin staining of distal ileal segments was performed at $6 \mathrm{~h}$ following sham burn or $30 \%$ TBSA burn. Data are representative of five independent experiments. Scale bar $=200 \mu \mathrm{m}$. (C) Immunoblot was performed to determine the expression of ZO-1, occludin, and claudin-1, with $\beta$-actin as loading control. Data represent mean \pm SEM. ${ }^{*} p<0.05,{ }^{* *} p<0.01$, compared with sham-burn group $(n=5)$. ${ }^{\#} p<0.05$, \#\# $p<0.01$, compared with burn group ( $\left.n=5\right)$. (D) ZO-1 was stained by immunofluorescence assay. Data are representative of five independent experiments. Scale bar $=20.0 \mu \mathrm{m}$.

can be seen in an obvious thinning and discontinuity of staining. However, 3-MA treatment did not obviously affect the morphological distribution of ZO-1 in sham-burned mice, but evidently alleviated the severe burn-induced relocalization of ZO-1.

\section{DISCUSSION}

The intestinal epithelial barrier plays an essential role in maintaining intestinal homeostasis. Burn injury, as one of the most common traumas, results in a mesenteric vasoconstriction and a lack of oxygen to the gut. Severe burn-induced damage participates in the occurrence and progress of gut barrier dysfunction and can activate the host's innate immune response. Thus, it results in the failure of the mucosa to act as an efficient barrier against luminal toxins, pathogenic organisms, and antigenic molecules (Saadia et al., 1990). Our previous studies revealed that severe burn increased the intestinal paracellular permeability to $4.4 \mathrm{kDa}$ FITC-dextran, caused histological damage of intestinal mucosa, and altered TJ protein expression and ZO-1 localization in mice (Chen et al., 2012; Huang et al., 2018), indicating that severe burn can induce the intestinal TJ barrier disruption. However, the underlying molecular mechanisms have not been fully elucidated so far.

Severe burn injury of the skin can induce intestinal dysfunction and pathology, including stress ulcers and increased intestinal 
permeability (Deitch, 1990; Epstein et al., 1991). Previous studies have reported that severe burn injury results in a significant reduction in blood flow to the small intestine (Kregel et al., 1988), which further leads to intestinal mucosal barrier dysfunction and induced ischemia at the villus tip (Simon, 1994; Hall et al., 2001; Yu et al., 2010). Intestinal ischemia causes the damage of intestinal mucosa (Moriwaki et al., 1993).

The process of protein folding is especially sensitive to endogenous or exogenous stress. The accumulation of unfolded proteins in the ER causes ER stress and induces the UPR, which alleviates stress through upregulating protein folding and degradation pathways in the ER inhibiting protein synthesis (Rutkowski and Kaufman, 2004; Schroder and Kaufman, 2005). Severe burninduced intestinal ischemia leads to oxidative stress and insufficient exogenous blood supply, resulting in limited nutrient delivery, and could induce further ER stress. Severe burn has been documented to induce ER stress in some visceral organs, suggesting that ER stress is an important cellular response to burn injury and is associated with organ damage and dysfunction (Gauglitz et al., 2010; Jeschke et al., 2012; Song et al., 2014). ER stress was reported to be associated with intestinal epithelial dysfunction in inflammatory bowel disease (Kaser et al., 2010; Adolph et al., 2013; Cao, 2015; Luo and Cao, 2015; Chotikatum et al., 2018). Several clinical studies have also shown evidence for enhanced ER stress in the intestines of patients with inflammatory bowel disease (Hu et al., 2007; Shkoda et al., 2007; Kaser et al., 2008). Although the UPR is initially to compensate for damage, chronic ER stress and defective UPR impair intestinal homeostasis, which may finally induce intestinal inflammation (Cao, 2016).

The epithelial TJs that control paracellular permeability are complex structures composed of the TJ proteins, such as transmembrane proteins (e.g., occludin and claudins), and peripheral membrane proteins (e.g., ZO-1). The localization and interactions of $\mathrm{TJ}$ proteins are critical in maintaining intestinal barrier function. Thus, alterations in TJ proteins are associated with changes in intestinal permeability. A study reported that acrolein-induced intestinal epithelium dysfunction is associated with a decrease/ redistribution of TJ proteins and intestinal ER stress (Chen et al., 2017). We previously observed that intestinal barrier dysfunction was associated with intestinal ER stress in response to severe burn (Huang et al., 2018). However, the exact role of ER stress in the burn-induced intestinal barrier dysfunction needed to be determined. Thus, in this study, we further demonstrate that activating ER stress with specific inducers Tm or Tg significantly aggravates the severe burn-induced intestinal epithelial TJ barrier dysfunction, whereas suppressing ER stress with specific inhibitor 4-PBA markedly alleviates the intestinal epithelial TJ barrier dysfunction caused by severe burn. Therefore, it is strongly suggested that the burn-triggered ER stress is an important cellular mechanism involved in the intestinal epithelial TJ barrier dysfunction induced by severe burn injury.

Autophagy is stimulated during situations of hypoxia, viral infection, nutrient deprivation, and ER stress (Avivar-Valderas et al., 2011; Hart et al., 2012). Accumulating evidence shows that ER stress induces autophagy under the condition of excessive accumulation of unfolded or misfolded proteins. Our previous study has demonstrated that ER stress and autophagy are both associated with the intestinal barrier dysfunction caused by severe burn injury (Huang et al., 2018). However, the interplay between ER stress and the activation of autophagy in intestinal epithelial cells following severe burn injury remains unknown. Here in the present study, we show that severe burn injuryinduced ER stress results in the activation of autophagy via downregulation of the $\mathrm{PI} 3 \mathrm{~K} / \mathrm{AKT} / \mathrm{mTOR}$ signaling pathway in intestinal epithelial cells. This is consistent with the other studies revealing that activation of $\mathrm{PI} 3 \mathrm{~K} / \mathrm{AKT} / \mathrm{mTOR}$ results in excessive autophagy (Aki et al., 2003; Qin et al., 2014).

A basal level of autophagy is considered cytoprotective when compared with apoptosis (Boya et al., 2005). However, a high level of autophagy is induced under stress conditions, which can indeed facilitate cell death in specific contexts (Denton et al., 2015). Thus, the biological significance of autophagy is still controversial. Of course, the regulatory role of autophagy in epithelial barrier dysfunction also remains to be clarified. Recent studies have shown that autophagy is a cytoprotective response to damage of the intestinal tract (Zhang et al., 2017a,b). Similarly, autophagy induced by nutrient starvation has been reported to regulate the conductivity of small uncharged molecules and cations to enhance intestinal barrier dysfunction via targeting claudin-2 protein degradation in Caco-2 cells (Nighot et al., 2015). However, a recent study has reported that autophagy activation results in endothelial barrier dysfunction, and inhibiting autophagy protects against endothelial barrier dysfunction by suppressing cadherin disassembly and actin stress fiber formation (Slavin et al., 2018). In this study, we demonstrate that inducing autophagy with rapamycin leads to the intestinal barrier dysfunction in sham-burned mice and aggravates the intestinal barrier dysfunction in severely burned mice, whereas inhibiting autophagy with 3-MA attenuates the severe burn-induced intestinal barrier dysfunction. Thus, it is suggested that autophagy activation contributes to the intestinal barrier dysfunction induced by severe burn injury.

It has been well documented that TJ proteins are crucial to the maintenance of intestinal epithelial barrier function (Turner, 2009). In this study, we reveal that inducing autophagy by rapamycin distinctly exacerbates the burn-induced decrease of TJ proteins ZO-1, occludin, and claudin-1, and worsens the burn-induced ZO-1 relocalization. In contrast, inhibiting autophagy by 3-MA dampens the burn-induced reduction of ZO-1, occludin, and claudin-1, and alleviates the burninduced relocalization of $\mathrm{ZO}-1$. In accordance with our present findings, the latest studies have demonstrated that autophagy activation results in the reduction and redistribution of $\mathrm{TJ}$ proteins ZO-1, occludin, and claudin-5 in endothelial cells, leading to the endothelial barrier disruption (Chan et al., 2018; Zhang et al., 2018). Thus, when all this is taken together, it is implied that autophagy activation is involved in the burninduced intestinal TJ barrier dysfunction, at least in part, by inducing both reduction and reorganization of TJ proteins.

In summary, it is concluded that severe burn injury triggers ER stress in intestinal epithelial cells, which in turn, induces autophagy activation via inhibiting the $\mathrm{PI} 3 \mathrm{~K} / \mathrm{AKT} / \mathrm{mTOR}$ signaling pathway. The ER stress-induced activation of autophagy contributes to the severe burn-induced intestinal epithelial TJ barrier dysfunction 
by causing both reduction and reorganization of TJ proteins. These novel findings, for the first time, suggest that the ER stressautophagy axis is involved in the intestinal epithelial TJ barrier dysfunction induced by severe burn injury. Therefore, inhibition of either ER stress or autophagy may be helpful to restore the intestinal epithelial TJ barrier following severe burn injury.

\section{DATA AVAILABILITY}

The raw data supporting the conclusions of this manuscript will be made available by the authors, without undue reservation, to any qualified researcher.

\section{ETHICS STATEMENT}

The animal studies were approved by the Animal Care and Use Committee of the Third Military Medical University (Army Medical University), Chongqing, China. And all animal

\section{REFERENCES}

Adolph, T. E., Tomczak, M. F., Niederreiter, L., Ko, H. J., Bock, J., Martinez-Naves, E., et al. (2013). Paneth cells as a site of origin for intestinal inflammation. Nature. 503, 272-276. doi: 10.1038/nature12599

Aki, T., Yamaguchi, K., Fujimiya, T., and Mizukami, Y. (2003). Phosphoinositide 3-kinase accelerates autophagic cell death during glucose deprivation in the rat cardiomyocyte-derived cell line H9c2. Oncogene 22, 8529-8535. doi: 10.1038/sj.onc. 1207197

Arnott, I. D., Kingstone, K., and Ghosh, S. (2000). Abnormal intestinal permeability predicts relapse in inactive Crohn disease. Scand. J. Gastroenterol. 35, 1163-1169. doi: 10.1080/003655200750056637

Arrieta, M. C., Madsen, K., Doyle, J., and Meddings, J. (2009). Reducing small intestinal permeability attenuates colitis in the IL10 gene-deficient mouse. Gut 58, 41-48. doi: 10.1136/gut.2008.150888

Avivar-Valderas, A., Salas, E., Bobrovnikova-Marjon, E., Diehl, J. A., Nagi, C., Debnath, J., et al. (2011). PERK integrates autophagy and oxidative stress responses to promote survival during extracellular matrix detachment. Mol. Cell. Biol. 31, 3616-3629. doi: 10.1128/MCB.05164-11

Ayala, P., Montenegro, J., Vivar, R., Letelier, A., Urroz, P. A., Copaja, M., et al. (2012). Attenuation of endoplasmic reticulum stress using the chemical chaperone 4-phenylbutyric acid prevents cardiac fibrosis induced by isoproterenol. Exp. Mol. Pathol. 92, 97-104. doi: 10.1016/j.yexmp.2011.10.012

Boya, P., Gonzalez-Polo, R.-A., Casares, N., Perfettini, J.-L., Dessen, P., Larochette, N., et al. (2005). Inhibition of macroautophagy triggers apoptosis. Mol. Cell. Biol. 25, 1025-1040. doi: 10.1128/MCB.25.3.1025-1040.2005

Cao, S. S. (2015). Endoplasmic reticulum stress and unfolded protein response in inflammatory bowel disease. Inflamm. Bowel Dis. 21, 636-644. doi: 10.1097/ MIB.0000000000000238

Cao, S. S. (2016). Epithelial ER stress in Crohn's disease and ulcerative colitis. Inflamm. Bowel Dis. 22, 984-993. doi: 10.1097/MIB.0000000000000660

Chan, Y., Chen, W., Wan, W., Chen, Y., Li, Y., and Zhang, C. (2018). Abeta1-42 oligomer induces alteration of tight junction scaffold proteins via RAGEmediated autophagy in bEnd.3 cells. Exp. Cell Res. 369, 266-274. doi: 10.1016/j.yexcr.2018.05.025

Chen, C., Wang, P., Su, Q., Wang, S., and Wang, F. (2012). Myosin light chain kinase mediates intestinal barrier disruption following burn injury. PLoS One 7:e34946. doi: 10.1371/journal.pone.0034946

Chen, W. Y., Wang, M., Zhang, J., Barve, S. S., McClain, C. J., and Joshi-Barve, S. (2017). Acrolein disrupts tight junction proteins and causes endoplasmic reticulum stress-mediated epithelial cell death leading to intestinal barrier procedures were performed in adherence to protocols approved by the Ethics Committee of Southwest Hospital, Third Military Medical University (Army Medical University).

\section{AUTHOR CONTRIBUTIONS}

YH performed the experiments, drafted the manuscript, and prepared the figures. YW and YF drafted parts of the manuscript and prepared the figures. $\mathrm{PW}$ and $\mathrm{XH}$ performed parts of the experiments. HR and FW designed the experiments and revised the manuscript.

\section{FUNDING}

This work was supported by the National Natural Science Foundation of China (81471871). The funders had no role in study design, data collection and analysis, decision to publish, or preparation of the manuscript.

dysfunction and permeability. Am. J. Pathol. 187, 2686-2697. doi: 10.1016/j. ajpath.2017.08.015

Chotikatum, S., Naim, H. Y., and El-Najjar, N. (2018). Inflammation induced ER stress affects absorptive intestinal epithelial cells function and integrity. Int. Immunopharmacol. 55, 336-344. doi: 10.1016/j.intimp.2017.12.016

Clark, A., Imran, J., Madni, T., and Wolf, S. E. (2017). Nutrition and metabolism in burn patients. Burns Trauma 5:11. doi: 10.1186/s41038-017-0076-x

Cooney, R., Baker, J., Brain, O., Danis, B., Pichulik, T., Allan, P., et al. (2010). NOD2 stimulation induces autophagy in dendritic cells influencing bacterial handling and antigen presentation. Nat. Med. 16, 90-97. doi: 10.1038/ nm.2069

Deitch, E. A. (1990). Intestinal permeability is increased in burn patients shortly after injury. Surgery 77, 411-416.

Denton, D., Xu, T. Q., and Kumar, S. (2015). Autophagy as a pro-death pathway. Immunol. Cell Biol. 93, 35-42. doi: 10.1038/icb.2014.85

Dong, K., Li, H., Zhang, M., Jiang, S., Chen, S., Zhou, J., et al. (2015). Endoplasmic reticulum stress induces up-regulation of hepatic beta-Klotho expression through ATF4 signaling pathway. Biochem. Biophys. Res. Commun. 459, 300-305. doi: 10.1016/j.bbrc.2015.02.104

Dong, Z., Zhou, J., Zhang, Y., Chen, Y., Yang, Z., Huang, G., et al. (2017). Astragaloside-IV alleviates heat-induced inflammation by inhibiting endoplasmic reticulum stress and autophagy. Cell. Physiol. Biochem. 42, 824-837. doi: 10.1159/000478626

Earley, Z. M., Akhtar, S., Green, S. J., Naqib, A., Khan, O., Cannon, A. R. et al. (2015). Burn injury alters the intestinal microbiome and increases gut permeability and bacterial translocation. PLoS One 10:e0129996. doi: 10.1371/journal.pone.0129996

Epstein, M. D., Tchervenkov, J. I., Alexander, J. W., Johnson, J. R., and Vester, J. W. (1991). Increased gut permeability following burn trauma. Arch. Surg. 126, 198-200. doi: 10.1001/archsurg.1991.01410260086012

Feng, Y., Wang, Y., Wang, P., Huang, Y., and Wang, F. (2018). Short-chain fatty acids manifest stimulative and protective effects on intestinal barrier function through the inhibition of NLRP3 inflammasome and autophagy. Cell. Physiol. Biochem. 49, 190-205. doi: 10.1159/000492853

Gauglitz, G. G., Halder, S., Boehning, D. F., Kulp, G. A., Herndon, D. N., Barral, J. M., et al. (2010). Post-burn hepatic insulin resistance is associated with endoplasmic reticulum (ER) stress. Shock 33, 299-305. doi: 10.1097/ SHK.0b013e3181b2f439

Groulx, J. F., Khalfaoui, T., Benoit, Y. D., Bernatchez, G., Carrier, J. C., Basora, N., et al. (2012). Autophagy is active in normal colon mucosa. Autophagy 8, 893-902. doi: 10.4161/auto.19738 
Hale, A. N., Ledbetter, D. J., Gawriluk, T. R., and Rucker, E. B. (2013). Autophagy: regulation and role in development. Autophagy 9, 951-992. doi: 10.4161/ auto. 24273

Hall, D. M., Buettner, G. R., Oberley, L. W., Xu, L., Matthes, R. D., and Gisolfi, C. V. (2001). Mechanisms of circulatory and intestinal barrier dysfunction during whole body hyperthermia. Am. J. Physiol. Heart Circ. Physiol. 280, H509-H521. doi: 10.1152/ajpheart.2001.280.2.H509

Hart, L. S., Cunningham, J. T., Datta, T., Dey, S., Tameire, F., Lehman, S. L., et al. (2012). ER stress-mediated autophagy promotes Myc-dependent transformation and tumor growth. J. Clin. Invest. 122, 4621-4634. doi: 10.1172/JCI62973

Hiramatsu, N., Chiang, W. C., Kurt, T. D., Sigurdson, C. J., and Lin, J. H. (2015). Multiple mechanisms of unfolded protein response-induced cell death. Am. J. Pathol. 185, 1800-1818. doi: 10.1016/j.ajpath.2015.03.009

Hosoi, T., and Ozawa, K. (2009). Endoplasmic reticulum stress in disease: mechanisms and therapeutic opportunities. Clin. Sci. 118, 19-29. doi: 10.1042/ CS20080680

Hosomi, S., Kaser, A., and Blumberg, R. S. (2015). Role of endoplasmic reticulum stress and autophagy as interlinking pathways in the pathogenesis of inflammatory bowel disease. Curr. Opin. Gastroenterol. 31, 81-88. doi: 10.1097/ MOG.0000000000000144

Hoyer-Hansen, M., and Jaattela, M. (2007). Connecting endoplasmic reticulum stress to autophagy by unfolded protein response and calcium. Cell Death Differ. 14, 1576-1582. doi: 10.1038/sj.cdd.4402200

Hu, S., Ciancio, M. J., Lahav, M., Fujiya, M., Lichtenstein, L., Anant, S., et al. (2007). Translational inhibition of colonic epithelial heat shock proteins by IFN-gamma and TNF-alpha in intestinal inflammation. Gastroenterology 133, 1893-1904. doi: 10.1053/j.gastro.2007.09.026

Huang, Y., Feng, Y., Wang, Y., Wang, P., Wang, F., and Ren, H. (2018). Severe burn-induced intestinal epithelial barrier dysfunction is associated with endoplasmic reticulum stress and autophagy in mice. Front. Physiol. 9:441. doi: 10.3389/fphys.2018.00441

Jeschke, M. G., Finnerty, C. C., Herndon, D. N., Song, J., Boehning, D., Tompkins, R. G., et al. (2012). Severe injury is associated with insulin resistance, endoplasmic reticulum stress response, and unfolded protein response. Ann. Surg. 255, 370-378. doi: 10.1097/SLA.0b013e31823e76e7

Kaser, A., Lee, A. H., Franke, A., Glickman, J. N., Zeissig, S., Tilg, H., et al. (2008). XBP1 links ER stress to intestinal inflammation and confers genetic risk for human inflammatory bowel disease. Cell 134, 743-756. doi: 10.1016/j. cell.2008.07.021

Kaser, A., Martinez-Naves, E., and Blumberg, R. S. (2010). Endoplasmic reticulum stress: implications for inflammatory bowel disease pathogenesis. Curr. Opin. Gastroenterol. 26, 318-326. doi: 10.1097/MOG.0b013e32833a9ff1

Kim, S. R., Kim, D. I., Kang, M. R., Lee, K. S., Park, S. Y., Jeong, J. S., et al. (2013). Endoplasmic reticulum stress influences bronchial asthma pathogenesis by modulating nuclear factor kappaB activation. J Allergy Clin Immunol Pract 132, 1397-1408. doi: 10.1016/j.jaci.2013.08.041

Kouroku, Y., Fujita, E., Tanida, I., Ueno, T., Isoai, A., Kumagai, H., et al. (2007). ER stress (PERK/eIF2alpha phosphorylation) mediates the polyglutamine-induced LC3 conversion, an essential step for autophagy formation. Cell Death Differ. 14, 230-239. doi: 10.1038/sj.cdd.4401984

Kregel, K. C., Wall, P. T., and Gisolfi, C. V. (1988). Peripheral vascular responses to hyperthermia in the rat. J. Appl. Physiol. 64, 2582-2588. doi: 10.1152/ jappl.1988.64.6.2582

Luo, K., and Cao, S. S. (2015). Endoplasmic reticulum stress in intestinal epithelial cell function and inflammatory bowel disease. Gastroenterol. Res. Pract. 2015:328791. doi: 10.1155/2015/328791

Magnotti, L. J., and Deitch, E. A. (2005). Burns, bacterial translocation, gut barrier function, and failure. J. Burn Care Rehabil. 26, 383-391. doi: 10.1097/01. bcr.0000176878.79267.e8

Mazzon, E., Sturniolo, G. C., Puzzolo, D., Frisina, N., and Fries, W. (2002). Effect of stress on the paracellular barrier in the rat ileum. Gut 51, 507-513. doi: 10.1136/gut.51.4.507

Moriwaki, Y., Katamura, H., Hashimoto, K., Ichikawa, Y., and Sugiyama, M. (1993). The worsening of tissue damage due to the transient release during the ischemia of rat small intestine-the relation with reactive oxygen species and lipid peroxidation. Nihon. Geka. Gakkai. Zasshi. 94, 1017-1021.
Nighot, P. K., Hu, C. A., and Ma, T. Y. (2015). Autophagy enhances intestinal epithelial tight junction barrier function by targeting claudin-2 protein degradation. J. Biol. Chem. 290, 7234-7246. doi: 10.1074/jbc.M114.597492

Ogata, M., Hino, S. I., Saito, A., Morikawa, K., Kondo, S., Kanemoto, S., et al. (2006). Autophagy is activated for cell survival after endoplasmic reticulum stress. Mol. Cell. Biol. 26, 9220-9231. doi: 10.1128/MCB.01453-06

Ozcan, U., Yilmaz, E., Ozcan, L., Furuhashi, M., Vaillancourt, E., Smith, R. O., et al. (2006). Chemical chaperones reduce ER stress and restore glucose homeostasis in a mouse model of type 2 diabetes. Science 313, 1137-1140. doi: $10.1126 /$ science.1128294

Ozean, U., Ozcan, L., Yilmaz, E., Duvel, K., Sahin, M., Manning, B. D., et al. (2008). Loss of the tuberous sclerosis complex tumor suppressors triggers the unfolded protein response to regulate insulin signaling and apoptosis. Mol. Cell 29, 541-551. doi: 10.1016/j.moicel.2007.12.023

Qin, L., Wang, Z., Tao, L., and Wang, Y. (2014). ER stress negatively regulates AKT/TSC/mTOR pathway to enhance autophagy. Autophagy 6, 239-247. doi: 10.4161 /auto.6.2.11062

Rutkowski, D. T., and Kaufman, R. J. (2004). A trip to the ER: coping with stress. Trends Cell Biol. 14, 20-28. doi: 10.1016/j.tcb.2003.11.001

Saadia, R., Schein, M., MacFarlane, C., and Boffard, K. D. (1990). Gut barrier function and the surgeon. Br. J. Surg. 77, 487-492. doi: 10.1002/bjs.1800770505

Schroder, M., and Kaufman, R. J. (2005). ER stress and the unfolded protein response. Mutat. Res. 569, 29-63.

Shkoda, A., Ruiz, P. A., Daniel, H., Kim, S. C., Rogler, G., Sartor, R. B., et al. (2007). Interleukin-10 blocked endoplasmic reticulum stress in intestinal epithelial cells: impact on chronic inflammation. Gastroenterology 132, 190-207. doi: $10.1053 /$ j.gastro.2006.10.030

Simon, H. B. (1994). Hyperthermia and heatstroke. Hosp. Pract. 29, 78-80.

Slavin, S. A., Leonard, A., Grose, V., Fazal, F., and Rahman, A. (2018). Autophagy inhibitor 3-methyladenine protects against endothelial cell barrier dysfunction in acute lung injury. Am. J. Physiol. Lung Cell. Mol. Physiol. 314, L388-L396. doi: 10.1152/ajplung.00555.2016

Song, J., de Libero, J., and Wolf, S. E. (2014). Hepatic autophagy after severe burn in response to endoplasmic reticulum stress. J. Surg. Res. 187, 128-133. doi: $10.1016 /$ j.jss.2013.09.042

Turner, J. R. (2009). Intestinal mucosal barrier function in health and disease. Nat. Rev. Immunol. 9, 799-809. doi: 10.1038/nri2653

Umeda, K., Ikenouchi, J., Katahira-Tayama, S., Furuse, K., Sasaki, H., Nakayama, M., et al. (2006). ZO-1 and ZO-2 independently determine where claudins are polymerized in tight-junction strand formation. Cell 126, 741-754. doi: 10.1016/j.cell.2006.06.043

Wagner, F., Henningsen, B., Lederer, C., Eichenmuller, M., Godeke, J., Muller-Hocker, J., et al. (2012). Rapamycin blocks hepatoblastoma growth in vitro and in vivo implicating new treatment options in high-risk patients. Eur. J. Cancer 48, 2442-2450. doi: 10.1016/j.ejca.2011.12.032

Wildenberg, M. E., Vos, A. C., Wolfkamp, S. C., Duijvestein, M., Verhaar, A. P., Te Velde, A. A., et al. (2012). Autophagy attenuates the adaptive immune response by destabilizing the immunologic synapse. Gastroenterology 142, 1493-1503.e1496. doi: 10.1053/j.gastro.2012.02.034

Wirawan, E., Vanden Berghe, T., Lippens, S., Agostinis, P., and Vandenabeele, P. (2012). Autophagy: for better or for worse. Cell Res. 22, 43-61. doi: 10.1038/ cr.2011.152

Xiao, R., Teng, M., Zhang, Q., Shi, X. H., and Huang, Y. S. (2012). Myocardial autophagy after severe burn in rats. PLoS One 7:e39488. doi: 10.1371/journal. pone.0039488

Yorimitsu, T., Nair, U., Yang, Z. F., and Klionsky, D. J. (2006). Endoplasmic reticulum stress triggers autophagy. J. Biol. Chem. 281, 30299-30304. doi: 10.1074/jbc.M607007200

Yu, J., Yin, P., Yin, J., Liu, F., Zhu, X., Cheng, G., et al. (2010). Involvement of ERK1/2 signalling and growth-related molecules' expression in response to heat stress-induced damage in rat jejunum and IEC-6 cells. Int. J. Hyperth. 26, 538-555. doi: 10.3109/02656736.2010.481276

Zhang, S., An, Q., Wang, T., Gao, S., and Zhou, G. (2018). Autophagy- and MMP-2/9-mediated reduction and redistribution of ZO-1 contribute to hyperglycemia-increased blood-brain barrier permeability during early reperfusion in stroke. Neuroscience 377, 126-137. doi: 10.1016/j. neuroscience.2018.02.035 
Zhang, D. Y., Qiu, W., Jin, P., Wang, P., and Sun, Y. (2017b). Role of autophagy and its molecular mechanisms in mice intestinal tract after severe burn. $J$. Trauma Acute Care Surg. 83, 716-724. doi: 10.1097/ta.0000000000001624

Zhang, D., Qiu, W., Wang, P., Zhang, P., Zhang, F., Wang, P., et al. (2017a). Autophagy can alleviate severe burn-induced damage to the intestinal tract in mice. Surgery 162, 408-417. doi: 10.1016/j.surg.2017.04.003

Zhang, X., Yuan, Y., Jiang, L., Zhang, J., Gao, J., Shen, Z., et al. (2014). Endoplasmic reticulum stress induced by tunicamycin and thapsigargin protects against transient ischemic brain injury: involvement of PARK2dependent mitophagy. Autophagy 10, 1801-1813. doi: 10.4161/auto.32136
Conflict of Interest Statement: The authors declare that the research was conducted in the absence of any commercial or financial relationships that could be construed as a potential conflict of interest.

Copyright (c) 2019 Huang, Wang, Feng, Wang, He, Ren and Wang. This is an openaccess article distributed under the terms of the Creative Commons Attribution License (CC BY). The use, distribution or reproduction in other forums is permitted, provided the original author(s) and the copyright owner(s) are credited and that the original publication in this journal is cited, in accordance with accepted academic practice. No use, distribution or reproduction is permitted which does not comply with these terms. 